\title{
UTILIZACIÓN DE OXITETRACICLINA E HIDROCORTISONA PARA EVITAR ADHERENCIAS EN DISPOSITIVOS INTRAVAGINALES EN OVINOS
}

\author{
Sosa, J.L. ${ }^{1} ;$ Fernandez, G. ${ }^{1}$; Boggero, C. ${ }^{1}$; \\ TOFFOLI, G.D. ${ }^{2}$ ' LEVA, P.E. ${ }^{2}$ \& GARCIA, M.S. ${ }^{2}$
}

\begin{abstract}
RESUMEN
Los dispositivos intravaginales son utilizados para la inducción y sincronización de celos. Los mismos pueden producir adherencia en las paredes de la vagina. El objetivo del presente trabajo fue probar la eficiencia de la aplicación de antibióticos y corticoide conjuntamente con los dispositivos para evitar adherencias. La experiencia se realizó con 45 ovejas distribuidas en tres grupos: GC dispositivo sin tratamiento, T1 dispositivo más tetraciclina y T2 dispositivo más tetraciclina y corticoide. Siguiendo el protocolo se procedió al retiro de los mismos a los 12 días. Se realizó un test $\chi 2$ para evaluar la efectividad del tratamiento. Las ovejas del T2 no presentaron adherencias. En cambio, se observaron distintos grados de adherencias: en el T1 (53 \%) y en el GC (46\%). Se puede inferir que la utilización de Tetracicilina más hidrocortisona es eficiente para evitar la vaginitis y por ende la adherencia de los dispositivos y pérdida por disminución de preñeces posteriores al retiro de los dispositivos.
\end{abstract}

Palabras clave: esponjas vaginales, adherencias, tetraciclina, corticoide.

\begin{abstract}
Different chemotherapeutics to prevent adhesions in intravaginal devices in sheep.

Intravaginal devices are used for the induction and synchronization of estrus. They may produce adhesion on the walls of the vagina. The objective of this study was to test the efficiency of the use of oxitetraciclina and hidrocortisona in conjunction with intravaginal devices to prevent adhesions. The experiment was carried out with 45 sheep divided into three groups: GC with an untreated de-
\end{abstract}

1.-Facultad de Ciencias Veterinarias (UNL). (3080HOF) Esperanza, provincia de Santa Fe. 2.-Facultad de Ciencias Agrarias (UNL). (3080HOF) Esperanza, provincia de Santa Fe. Email: pleva@fca.unl.edu.ar

Manuscrito recibido el 19 de octubre de 2015 y aceptado para su publicación el 6 de septiembre de 2016. 


\section{J.L. Sosa et al.}

vice, $\mathrm{T} 1$ with a device plus tetracycline and $\mathrm{T} 2$ with a device plus tetracycline and corticosteroid. Following the protocol, devices were removed after 12 days. A test $\chi 2$ was conducted to assess treatment effectiveness. T2 sheep showed no adhesions. Instead, varying degrees of adhesion were observed in $\mathrm{T} 1(53 \%)$ and GC ( $46 \%)$. It can be inferred that the use of tetracycline plus corticosteroid is efficient to prevent vaginitis and therefore the adherence of devices and loss by diminishing post-removal pregnancies.

Key words: intravaginal devices, adherence, tetracycline, corticosteroids.

\section{INTRODUCCIÓN}

En los últimos años, la producción ovina y en especial la fabricación artesanal de quesos, resurgió debido al alto valor agregado de los mismos (Dulce, 2005). Este tipo de actividad se encuentra en franco crecimiento a lo largo y ancho del país en diferentes regiones y modalidades productivas.

Las ovejas (Ovis aries) son poliéstricas estacionales (Fernández Abella y Villegas, 1995) de modo que paren en la época más favorable del año, o sea en la primavera. Esta característica impide la obtención de un flujo continuo de materia prima para la industria.

Para poder mantener el flujo de materia prima es necesario manejar la estación reproductiva en el ovino y trasladar sistemas tradicionales a partos extemporáneos para, de este modo, proveer productos al mercado todo el año.

Ayuda a este propósito la manipulación de la reproducción ya que acorta el interparto y aumenta un parto cada dos años, así se mantiene casi constante el proceso de ordeño. De esta manera se induce un estro fuera de la temporada reproductiva. En la actualidad el método más utilizado en pequeños rumiantes para tal finalidad son los dispositivos intravaginales que liberan medroxiprogesterona, que simulan un cuerpo lúteo funcional y que al retirarlo se produce la iniciación del estro y, consecuentemente, la ovulación, luego de un período de tiempo en que la hembra está receptiva (Scudamore et al., 1993). Estos dispositivos se pueden utilizar, además, como metodología ya sea para realizar la inseminación artificial o para el servicio natural, a fin de conocer con exactitud la fecha de parto y la paternidad para el caso de realizar una selección. Es decir que se cuenta con una serie de elementos que nos permiten un manejo más apropiado, con todas las ventajas que de este procedimiento se desprende.

En oportunidades suelen romperse al tratarlos de desprender de la adherencia, y esto genera inconvenientes.

Algunos autores (Azzarini, 1995; Devincenzi et al., 1995) utilizaron dispositivos intravaginales con progesterona, esponjas (EIV) o CIDR (controlled internal drug release device), y lograron buenas respuestas y sin adherencias al retirar los mismos. Sus desventajas son la necesidad de espolvorearlas con antibiótico, debido a que muchas se adhieren a la pared vaginal por un proceso inflamatorio local provocado por el propio dispositivo que actúa como un cuerpo extraño y por una vaginitis leve (Bonino et al., 1989; Bonino, 1995). 